\title{
NPSR1 Gene Is Associated with Reduced Risk of Rheumatoid Arthritis
}

\author{
GEMA ROBLEDO, MIGUEL ANGEL GONZÁLEZ-GAY, BENJAMÍN FERNÁNDEZ-GUTIÉRREZ, \\ JOSÉ RAMÓN LAMAS, ALEJANDRO BALSA, DORA PASCUAL-SALCEDO, SANTOS CASTAÑEDA, \\ RICARDO BLANCO, ISIDORO GONZÁLEZ-ALVARO, ANTONIO GARCÍA, ENRIQUE RAYA, \\ CARMEN GÓMEZ-VAQUERO, MARIO DELGADO, and JAVIER MARTÍN
}

\begin{abstract}
Objective. Neuropeptide S receptor 1 (NPSRl) is a G protein-coupled receptor involved in immune response and is associated with several inflammatory diseases. We investigated the possible contribution of several polymorphisms in the intronic region of NPSR1 to rheumatoid arthritis (RA).

Methods. Genotyping of 7 single-nucleotide polymorphisms (SNP) was performed in a total of 1232 patients with RA and 983 healthy controls of Spanish white origin by real-time polymerase chain reaction technology, using the TaqMan 5'-allele discrimination assay.

Results. One out of the 7 SNP analyzed (rs740347) was associated with RA [p after Bonferroni correction $\left(\mathrm{p}_{\mathrm{BNF}}\right)=1.2 \times 10^{-3}$, OR 0.73]. An association was also observed with rheumatoid factor-positive and shared epitope-positive RA ( $\mathrm{p}_{\mathrm{BNF}}=0.011$, OR $0.73 ; \mathrm{p}_{\mathrm{BNF}}=0.037, \mathrm{OR} 0.75$, respectively).

Conclusion. Our results show that variations in the NPSR1 intronic region are associated with low risk in patients with RA, supporting other evidence that this locus represents a common genetic factor in inflammatory diseases. (First Release May 1 2012; J Rheumatol 2012;39:1166-70; doi:10.3899/ jrheum.111205)
\end{abstract}

Key Indexing Terms:

RHEUMATOID ARTHRITIS NPSR1 RHEUMATOID FACTOR SHARED EPITOPE ANTICITRULLINATED PROTEIN ANTIBODIES SINGLE-NUCLEOTIDE POLYMORPHISM

Rheumatoid arthritis (RA) is a chronic systemic autoimmune disease, characterized by inflammation of synovial joints and pain affecting approximately $1 \%$ of the population worldwide.

From the Instituto de Parasitología y Biomedicina López-Neyra, CSIC, Granada; Department of Rheumatology, Hospital Universitario Marqués de Valdecilla, IFIMAV, Santander; Department of Rheumatology, Hospital Clínico San Carlos, Madrid; Department of Rheumatology, Hospital Universitario La Paz, Madrid; Department of Rheumatology, Hospital Universitario La Princesa, Madrid; Department of Rheumatology, Hospital Virgen de las Nieves, Granada; Department of Rheumatology, Hospital Universitario Clínico San Cecilio, Granada; and Department of Rheumatology, Hospital Universitario de Bellvitge, IDIBELL,

L'Hospitalet de Llobregat, Barcelona, Spain.

Supported by a grant from RETICS Program, RD08/0075 (RIER), from Instituto de Salud Carlos III (ISCIII), Spain. Supported also in part by a European IMI BTCure Program grant.

G. Robledo, PhD; M. Delgado, PhD; J. Martín, MD, PhD, Instituto de Parasitología y Biomedicina López-Neyra, CSIC; M.A. González-Gay, $M D, P h D ; R$. Blanco, MD, PhD, Department of Rheumatology, Hospital Universitario Marqués de Valdecilla, IFIMAV; B. Fernández-Gutiérrez, MD, PhD; J.R. Lamas, PhD, Department of Rheumatology, Hospital Clínico San Carlos; A. Balsa, MD, PhD; D. Pascual-Salcedo, PhD, Department of Rheumatology, Hospital Universitario La Paz;

S. Castañeda, MD, PhD; I. González-Alvaro, $M D$, PhD, Department of Rheumatology, Hospital Universitario La Princesa; A. García, MD, PhD, Department of Rheumatology, Hospital Virgen de las Nieves; E. Raya, $M D, P h D$, Department of Rheumatology, Hospital Universitario Clínico San Cecilio; C. Gómez-Vaquero, MD, PhD, Department of Rheumatology, Hospital Universitario de Bellvitge, IDIBELL, L'Hospitalet del Llobregat.

Address correspondence to Dr. J. Martín, Instituto de Parasitología y Biomedicina López-Neyra, CSIC, Parque Tecnológico de Ciencias de la Salud, Avenida del Conocimiento s/n 18100, Armilla, Granada, Spain. E-mail:martin@ipb.csic.es

Accepted for publication March 7, 2012.
Both genetic and environmental factors seem to be involved in its complex etiology, although a strong genetic component has been described ${ }^{1,2}$.

Neuropeptide S receptor 1 (NPSRI), located in chromosome $7 \mathrm{p}$, is a G-protein coupled receptor gene involved in leukocyte immune response ${ }^{3}$. Several studies have shown that NPSR1 expression is upregulated in inflammatory processes such as asthma, inflammatory bowel disease (IBD), and intestinal and cutaneous inflammatory diseases ${ }^{4,5,6}$, suggesting a main role of this gene in inflammation.

We analyzed 7 variations along the NPSRI gene in RA patients and controls from Spain. Five of them (rs323917, rs323922, rs324377, rs324396, and rs740347) are located at the region between exons 2 and 3 of the NPSRl gene and have been previously published as conferring genetic risk for diseases that present chronic inflammation including asthma ${ }^{4,7,8}$ and $\mathrm{IBD}^{5,9}$. Further, 2 additional single-nucleotide polymorphisms (SNP) (rs324987 and rs10263447) located in introns 3 and 4, respectively, were included in this study. Recently, these variations were reported to be associated with anticitrullinated protein antibody (ACPA)-negative RA and with disease activity in a Swedish population ${ }^{10}$.

\section{MATERIALS AND METHODS}

Patients. Our case-control study consisted of 1232 Spanish white patients with RA who met the American College of Rheumatology revised criteria for RA $^{11}$ and 983 blood donors. All samples were matched by age and sex.

In our cohort $73.1 \%$ of the samples were characterized for rheumatoid factor (RF), $65.4 \%$ for shared epitope (SE), and $63.2 \%$ for ACPA. Of these

Personal non-commercial use only. The Journal of Rheumatology Copyright @ $\odot 2012$. All rights reserved. 
patients, $67.6 \%$ were positive for RF, $65.4 \%$ encoded the SE, and $59.3 \%$ were seropositive for ACPA. All patients and controls were enrolled with local ethics committee approval and provided their informed consent.

NPSR1 SNP selection and genotyping. For genotyping, genomic DNA from patients and controls was isolated from peripheral white blood cells, using standard procedures. Seven SNP (dbSNP rs323917, rs323922, rs324377, rs324396, rs740347, rs324987, and rs10263447) localized in the NPSR1 gene were selected for investigation. Samples were genotyped using TaqMan ${ }^{\circledR} 5$, allelic discrimination Assay-By-Design method. The genotype call rate for every SNP was $>94.5 \%$ in controls and $>93.9 \%$ in patients.

Statistical analysis. All statistical analyses of the allele frequencies were carried out using the Linux software PLINK v1.07 (http://pngu.mgh.harvard. edu/purcell/plink/). To test for association, $2 \times 2$ contingency tables and chisquare and/or Fisher's exact tests, when appropriate, were used to calculate $\mathrm{p}$ values, OR, and 95\% CI. Allelic combinations were analyzed using Haploview (V.4.2) and Unphased.

\section{RESULTS}

We performed single-point association analysis of 7 representative SNP, along the intronic region of NPSR1 (for details, see Appendix). All the SNP showed allele frequencies similar to those previously reported in European populations ${ }^{4,5}$. In a first step, we analyzed the possible implication of these SNP in RA susceptibility (Table 1, upper rows). We found an association for rs740347. The minor allele (C) was significantly decreased in RA patients compared to healthy controls [p after Bonferroni correction $\left(\mathrm{p}_{\mathrm{BNF}}\right)=1.2 \mathrm{E}-03$; OR 0.73].

To assess the possible implications of these 7 SNP for RA severity, we performed a stratification analysis for the presence of RF, SE alleles, and ACPA, when available (Table 1, lower rows). We found an association when comparing patients positive for RF or SE with controls for the NPSR1 rs740347: RF ( $\left.\mathrm{p}_{\mathrm{BNF}}=0.011 ; \mathrm{OR} 0.73\right), \mathrm{SE}\left(\mathrm{p}_{\mathrm{BNF}}=0.037\right.$; OR $0.75)$. When we compared patients negative for RF, SE, or ACPA with controls, no association was observed for any of the 7 SNP (data not shown). Additionally, we compared RA patients positive for RF, SE, and ACPA with RA patients negative for each (Table 2). No association was detected for any of the analyzed SNP. This suggests that rs740347 is not associated with a clinical phenotype but with the global disease.

Table 3 shows allelic combination tests performed to analyze the possible interaction between the SNP of this genomic region. We found 3 different allelic combinations associated with RA susceptibility (CGCCGGC and CGCTGTG) or protection (CCACCGC).

\section{DISCUSSION}

NPSRl gene seems to play a role in asthma ${ }^{7,8}, \mathrm{IBD}^{5,9}$, and $\mathrm{RA}^{10}$ in independent studies with ethnically different populations.

One of the 7 SNP analyzed in this study, rs740347, showed strong evidence of association with RA after correction for multiple comparisons. The rs $740347 \mathrm{C}$ allele conferred a protective effect on the global disease. On the other hand, the allelic combination analysis of NPSRl variants suggests that the genomic region of this gene plays an important role in RA association.
The presence of positive RF, ACPA, and HLA-DRB1 alleles encoding a conserved sequence at amino acid positions 70-74, commonly known as the SE, has been associated with susceptibility and severity of RA ${ }^{12,13}$. We performed a stratification comparing patients with RA who were positive and negative for SE, RF, and ACPA with each other and with controls. We found an association for rs740347 comparing RF-and SE-positive patients with controls but not with RF-and SE-negative patients. It may be speculated that rs740347 is linked to the global disease and not to a subtype. The minor allele (C) of this SNP was previously seen to confer susceptibility to asthma in German and Italian populations ${ }^{7,14}$, but showed a low risk for susceptibility in a Colombian cohort $^{15}$, similar to our results. Additionally, D'Amato, et al analyzed 19 NPSR 1 polymorphisms in a RA cohort from Sweden ${ }^{10}$. However, none of the SNP analyzed showed association with the global disease. Population genetic variability between the 2 cohorts or interactions with other genes may be responsible for the lack of coincidence in the results. This group showed that rs324987 and rs10263447 were associated with ACPA-negative RA and disease activity. In our population these $2 \mathrm{SNP}$ showed no evidence of association with the disease or with any of the subgroups analyzed.

Intronic position of the studied SNP suggests a possible involvement of associated polymorphism in regulation of splicing and protein isoform production. Variations in rs740347 may downregulate the function of NPSR1 in immunomodulation.

Our results provide evidence of involvement of NPSR 1 in the genetic contribution for RA association in a Spanish population. Other replication studies and functional analyses are necessary to better elucidate its role in chronic inflammatory diseases.

\section{ACKNOWLEDGMENT}

The authors thank Sofía Vargas and Sonia Rodríguez (Instituto de Parasitología y Biomedicina López-Neyra, CSIC, Spain) for their excellent technical assistance; and all the patients and healthy controls for their essential collaboration.

\section{REFERENCES}

1. Orozco G, Rueda B, Martin J. Genetic basis of rheumatoid arthritis. Biomed Pharmacother 2006;60:656-62.

2. Scott DL, Wolfe F, Huizinga TW. Rheumatoid arthritis. Lancet 2010;376:1094-108.

3. Pulkkinen V, Majuri ML, Wang G, Holopainen P, Obase Y, Vendelin $\mathrm{J}$, et al. Neuropeptide $\mathrm{S}$ and $\mathrm{G}$ protein-coupled receptor 154 modulate macrophage immune responses. Hum Mol Genet 2006;15:1667-79.

4. Laitinen T, Polvi A, Rydman P, Vendelin J, Pulkkinen V, Salmikangas $\mathrm{P}$, et al. Characterization of a common susceptibility locus for asthma-related traits. Science 2004;304:300-4.

5. D'Amato M, Bruce S, Bresso F, Zucchelli M, Ezer S, Pulkkinen V, et al. Neuropeptide s receptor 1 gene polymorphism is associated with susceptibility to inflammatory bowel disease. Gastroenterology 2007;133:808-17.

6. Sundman L, Saarialho-Kere U, Vendelin J, Lindfors K, Assadi G, Kaukinen $\mathrm{K}$, et al. Neuropeptide $\mathrm{S}$ receptor 1 expression in the

Personal non-commercial use only. The Journal of Rheumatology Copyright @ 2012. All rights reserved. 
Table 1. Genotype and minor allele frequencies of SNP located in the NPSR1 gene in patients with rheumatoid arthritis and healthy controls from Spain, according to specific characteristics of the disease. Values shown in bold type indicate statistical significance.

\begin{tabular}{|c|c|c|c|c|c|c|c|c|c|}
\hline \multirow[b]{2}{*}{ SNP } & \multirow[b]{2}{*}{$1 / 2$} & \multirow[b]{2}{*}{ Subgroup (N) } & \multicolumn{3}{|c|}{ Genotype, n (\%) } & \multirow[b]{2}{*}{ MAF (\%) } & \multicolumn{3}{|c|}{ Allele Test } \\
\hline & & & $1 / 1$ & $1 / 2$ & $2 / 2$ & & $p^{*}$ & $p_{\mathrm{BNF}} * *$ & OR (CI 95\%]*** \\
\hline \multirow[t]{5}{*}{ rs323917 } & $\mathrm{C} / \mathrm{G}$ & Controls $(n=945)$ & $859(90.90)$ & $78(8.25)$ & $8(0.85)$ & 4.97 & & & \\
\hline & & $\mathrm{RA}(\mathrm{n}=1166)$ & $1041(89.28)$ & $116(9.97)$ & $9(0.75)$ & 5.75 & 0.269 & 0.999 & $1.17(0.88-1.55)$ \\
\hline & & $R F+(n=584)$ & $512(87.67)$ & $67(11.47)$ & $5(0.86)$ & 6.59 & 0.057 & 0.399 & $1.36(0.99-1.86)$ \\
\hline & & $\mathrm{SE}+(\mathrm{n}=508)$ & $453(89.17)$ & $50(9.84)$ & $5(0.98)$ & 5.91 & 0.275 & 0.999 & $1.21(0.86-1.69)$ \\
\hline & & $\mathrm{ACPA}+(\mathrm{n}=453)$ & $404(89.18)$ & $45(9.93)$ & $4(0.88)$ & 5.85 & 0.320 & 0.999 & $1.20(0.84-1.70)$ \\
\hline \multirow[t]{5}{*}{ rs323922 } & $\mathrm{G} / \mathrm{C}$ & Controls $(n=929)$ & $306(32.94)$ & $504(54.25)$ & $109(11.73)$ & 39.86 & & & \\
\hline & & $\mathrm{RA}(\mathrm{n}=1157)$ & $436(37.68)$ & $573(49.52)$ & $148(12.80)$ & 37.55 & 0.158 & 0.999 & $0.91(0.80-1.04)$ \\
\hline & & $R F+(n=582)$ & $213(36.60)$ & $296(50.86)$ & $73(12.54)$ & 37.97 & 0.323 & 0.999 & $0.93(0.79-1.08)$ \\
\hline & & $\mathrm{SE}+(\mathrm{n}=504)$ & $181(35.91)$ & $252(50.00)$ & $71(14.09)$ & 39.09 & 0.712 & 0.999 & $0.97(0.83-1.14)$ \\
\hline & & $\mathrm{ACPA}+(\mathrm{n}=443)$ & $164(37.02)$ & $219(49.44)$ & $60(13.54)$ & 38.26 & 0.445 & 0.999 & $0.94(0.79-1.11)$ \\
\hline \multirow[t]{5}{*}{ rs 324377} & $\mathrm{C} / \mathrm{A}$ & Controls $(n=945)$ & $332(35.13)$ & $452(47.83)$ & $161(17.04)$ & 40.95 & & & \\
\hline & & $\mathrm{RA}(\mathrm{n}=1182)$ & $411(34.77)$ & $575(48.65)$ & $196(16.58)$ & 40.90 & 0.976 & 0.999 & $1.00(0.88-1.13)$ \\
\hline & & $R F+(n=595)$ & $204(34.29)$ & $292(49.08)$ & $99(16.64)$ & 41.18 & 0.909 & 0.999 & $1.01(0.87-1.17)$ \\
\hline & & $\mathrm{SE}+(\mathrm{n}=516)$ & $168(32.56)$ & $259(50.19)$ & $89(17.25)$ & 42.34 & 0.475 & 0.999 & $1.06(0.91-1.24)$ \\
\hline & & $\mathrm{ACPA}+(\mathrm{n}=460)$ & $159(34.57)$ & $218(47.39)$ & $83(18.04)$ & 41.74 & 0.699 & 0.999 & $1.03(0.88-1.21)$ \\
\hline \multirow[t]{5}{*}{ rs 324396} & $\mathrm{C} / \mathrm{T}$ & Controls $(\mathrm{n}=951)$ & $439(46.16)$ & $399(41.96)$ & $113(11.88)$ & 32.86 & & & \\
\hline & & $\mathrm{RA}(\mathrm{n}=1157)$ & $547(47.28)$ & $480(41.49)$ & $130(11.23)$ & 32.00 & 0.585 & 0.999 & $0.96(0.84-1.10)$ \\
\hline & & $\mathrm{RF}+(\mathrm{n}=581)$ & $274(47.16)$ & $240(41.31)$ & $67(11.53)$ & 32.19 & 0.710 & 0.999 & $0.97(0.83-1.14)$ \\
\hline & & $\mathrm{SE}+(\mathrm{n}=503)$ & $243(48.31)$ & $206(40.95)$ & $54(10.74)$ & 31.21 & 0.377 & 0.999 & $0.93(0.79-1.10)$ \\
\hline & & $\mathrm{ACPA}+(\mathrm{n}=445)$ & $214(48.09)$ & $185(41.57)$ & $46(10.34)$ & 31.12 & 0.370 & 0.999 & $0.92(0.78-1.10)$ \\
\hline \multirow[t]{5}{*}{ rs740347 } & $\mathrm{G} / \mathrm{C}$ & Controls $(\mathrm{n}=952)$ & $614(64.50)$ & $311(32.67)$ & $27(2.83)$ & 19.17 & & & \\
\hline & & $\mathrm{RA}(\mathrm{n}=1166)$ & $848(72.73)$ & $290(24.87)$ & $28(2.40)$ & 14.84 & $1.7 \mathrm{E}-04$ & $1.2 \mathrm{E}-03$ & $0.73(0.62-0.86)$ \\
\hline & & $R F+(n=609)$ & $446(73.23)$ & $146(23.97)$ & $17(2.79)$ & 14.78 & $1.6 \mathrm{E}-03$ & 0.011 & $0.73(0.60-0.89)$ \\
\hline & & $\mathrm{SE}+(\mathrm{n}=527)$ & $381(72.30)$ & $133(25.24)$ & $13(2.47)$ & 15.09 & $5.3 \mathrm{E}-03$ & 0.037 & $0.75(0.61-0.92)$ \\
\hline & & $\mathrm{ACPA}+(\mathrm{n}=462)$ & $326(70.56)$ & $120(25.97)$ & $16(3.46)$ & 16.45 & 0.079 & 0.553 & $0.83(0.68-1.02)$ \\
\hline \multirow[t]{5}{*}{ rs324987 } & $\mathrm{T} / \mathrm{C}$ & Controis $(\mathrm{n}=943$ ) & $334(35.42)$ & $452(47.93)$ & $157(16.65)$ & 40.62 & & & \\
\hline & & $\mathrm{RA}(\mathrm{n}=1161)$ & $420(36.18)$ & $539(46.42)$ & $202(17.40)$ & 40.61 & 0.996 & 0.999 & $1.00(0.88-1.14)$ \\
\hline & & $R F+(n=591)$ & $214(36.21)$ & $276(46.70)$ & $101(17.09)$ & 40.44 & 0.929 & 0.999 & $0.99(0.85-1.15)$ \\
\hline & & $\mathrm{SE}+(\mathrm{n}=518)$ & $176(33.98)$ & $255(49.23)$ & $87(16.80)$ & 41.41 & 0.676 & 0.999 & $1.03(0.88-1.21)$ \\
\hline & & $\mathrm{ACPA}+(\mathrm{n}=459)$ & $154(33.55)$ & $228(49.67)$ & $77(16.78)$ & 41.61 & 0.615 & 0.999 & $1.04(0.89-1.23)$ \\
\hline \multirow[t]{5}{*}{ rs 10263447} & $\mathrm{G} / \mathrm{C}$ & Controls $(\mathrm{n}=955)$ & $719(75.29)$ & $214(22.41)$ & $22(2.30)$ & 13.51 & & & \\
\hline & & $\mathrm{RA}(\mathrm{n}=1167)$ & $860(73.69)$ & $279(23.91)$ & $28(2.40)$ & 14.35 & 0.451 & 0.999 & $1.07(0.89-1.29)$ \\
\hline & & $R F+(n=593)$ & $438(73.86)$ & $141(23.78)$ & $14(2.36)$ & 14.25 & 0.583 & 0.999 & $1.06(0.86-1.31)$ \\
\hline & & $\mathrm{SE}+(\mathrm{n}=520)$ & $387(74.42)$ & $120(23.08)$ & $13(2.50)$ & 14.04 & 0.710 & 0.999 & $1.04(0.84-1.30)$ \\
\hline & & $\mathrm{ACPA}+(\mathrm{n}=460)$ & $344(74.78)$ & $106(23.04)$ & $10(2.17)$ & 13.70 & 0.911 & 0.999 & $1.01(0.80-1.28)$ \\
\hline
\end{tabular}

${ }^{*}$ All $\mathrm{p}$ values have been calculated for the allelic model; ${ }^{* *}$ p-values after Bonferroni correction for 7 tests; ${ }^{* * *}$ Odds ratio for the minor allele. $\mathrm{RF}+/$ : positive/negative rheumatoid factor; $\mathrm{SE}+/-:$ positive/negative shared epitope; ACPA+/-: positive/negative anticitrullinated protein antibody. MAF: minor allele frequency.

intestine and skin - putative role in peptide hormone secretion.

Neurogastroenterol Motil 2010;22:79-87, e30.

7. Malerba G, Lindgren CM, Xumerle L, Kiviluoma P, Trabetti E,
Laitinen T, et al. Chromosome 7p linkage and GPR154 gene association in Italian families with allergic asthma. Clin Exp Allergy 2007;37:83-9. 
Table 2. Genotype and minor allele frequencies of SNP located in the NPSR1 gene, comparing positive versus negative rheumatoid factor, positive versus negative shared epitope, and positive versus negative anticitrullinated peptide antibody in patients with rheumatoid arthritis from Spain.

\begin{tabular}{|c|c|c|c|c|c|c|c|c|c|}
\hline \multirow[b]{2}{*}{ SNP } & \multirow[b]{2}{*}{$1 / 2$} & \multicolumn{4}{|c|}{ Genotype, n (\%) } & \multirow[b]{2}{*}{ MAF (\%) } & \multicolumn{3}{|c|}{ Allele Test } \\
\hline & & Subgroup (N) & $1 / 1$ & $1 / 2$ & $2 / 2$ & & $\mathrm{p}^{*}$ & $\mathrm{p}_{\mathrm{BNF}}{ }^{* *}$ & OR (CI 95\%)*** \\
\hline \multirow[t]{6}{*}{ rs323917 } & $\mathrm{C} / \mathrm{G}$ & RF- $(n=290)$ & $262(90.34)$ & $26(8.97)$ & $2(0.69)$ & 5.17 & & & \\
\hline & & $R F+(n=584)$ & $512(87.67)$ & $67(11.47)$ & $5(0.86)$ & 6.59 & 0.244 & 0.999 & $1.29(0.84-2.00)$ \\
\hline & & SE- $(n=276)$ & $242(87.68)$ & $31(11.23)$ & $3(1.09)$ & 6.7 & & & \\
\hline & & $\mathrm{SE}+(\mathrm{n}=508)$ & $453(89.17)$ & $50(9.84)$ & $5(0.98)$ & 5.9 & 0.531 & 0.999 & $0.87(0.57-1.33)$ \\
\hline & & ACPA- $(n=307)$ & $270(87.95)$ & $36(11.73)$ & $1(0.33)$ & 6.18 & & & \\
\hline & & $\mathrm{ACPA}+(\mathrm{n}=453)$ & $404(89.18)$ & $45(9.93)$ & $4(0.88)$ & 5.85 & 0.785 & 0.999 & $0.94(0.61-1.45)$ \\
\hline \multirow[t]{6}{*}{ rs323922 } & $\mathrm{G} / \mathrm{C}$ & $R F-(n=290)$ & $114(39.31)$ & $138(47.59)$ & $38(13.10)$ & 36.9 & & & \\
\hline & & $R F+(n=582)$ & $213(36.60)$ & $296(50.86)$ & $73(12.54)$ & 37.97 & 0.662 & 0.999 & $1.05(0.85-1.29)$ \\
\hline & & SE- $(n=279)$ & $115(41.22)$ & $132(47.31)$ & $32(11.47)$ & 35.13 & & & \\
\hline & & $\mathrm{SE}+(\mathrm{n}=504)$ & $181(35.91)$ & $252(50.00)$ & $71(14.09)$ & 39.09 & 0.121 & 0.847 & $1.19(0.96-1.47)$ \\
\hline & & ACPA- $(n=311)$ & $120(38.59)$ & $157(50.48)$ & $34(10.93)$ & 36.17 & & & \\
\hline & & $\mathrm{ACPA}+(\mathrm{n}=443)$ & $164(37.02)$ & $219(49.44)$ & $60(13.54)$ & 38.26 & 0.409 & 0.999 & $1.09(0.88-1.35)$ \\
\hline \multirow[t]{6}{*}{ rs324377 } & $\mathrm{C} / \mathrm{A}$ & $R F-(n=294)$ & 99 (33.67) & $146(49.66)$ & $49(16.67)$ & 41.5 & & & \\
\hline & & $R F+(n=595)$ & $204(34.29)$ & $292(49.08)$ & $99(16.64)$ & 41.18 & 0.897 & 0.999 & $0.99(0.81-1.21)$ \\
\hline & & SE- $(n=277)$ & $105(37.91)$ & $124(44.77)$ & $48(17.33)$ & 39.71 & & & \\
\hline & & $\mathrm{SE}+(\mathrm{n}=516)$ & $168(32.56)$ & $259(50.19)$ & $89(17.25)$ & 42.34 & 0.310 & 0.999 & $1.12(0.90-1.38)$ \\
\hline & & ACPA- $(n=316)$ & $116(36.71)$ & $151(47.78)$ & $49(15.51)$ & 39.4 & & & \\
\hline & & $\mathrm{ACPA}+(\mathrm{n}=460)$ & $159(34.57)$ & $218(47.39)$ & $83(18.04)$ & 41.74 & 0.357 & 0.999 & $1.10(0.90-1.36)$ \\
\hline \multirow[t]{6}{*}{ rs324396 } & $\mathrm{T} / \mathrm{C}$ & RF- $(n=293)$ & $137(46.76)$ & $123(41.98)$ & $33(11.26)$ & 32.25 & & & \\
\hline & & $R F+(n=581)$ & $274(47.16)$ & $240(41.31)$ & $67(11.53)$ & 32.19 & 0.978 & 0.999 & $1.00(0.81-1.23)$ \\
\hline & & SE- $(n=274)$ & $125(45.62)$ & $118(43.07)$ & $31(11.31)$ & 32.85 & & & \\
\hline & & $\mathrm{SE}+(\mathrm{n}=503)$ & $243(48.31)$ & $206(40.95)$ & $54(10.74)$ & 31.21 & 0.509 & 0.999 & $0.93(0.74-1.16)$ \\
\hline & & ACPA- $(n=310)$ & $135(43.55)$ & $135(43.55)$ & $40(12.90)$ & 34.68 & & & \\
\hline & & $\mathrm{ACPA}+(\mathrm{n}=445)$ & $214(48.09)$ & $185(41.57)$ & $46(10.34)$ & 31.12 & 0.147 & 0.999 & $0.85(0.68-1.06)$ \\
\hline \multirow[t]{6}{*}{ rs 740347} & $\mathrm{G} / \mathrm{C}$ & $R F-(n=292)$ & $203(69.52)$ & $80(27.40)$ & $9(3.08)$ & 16.78 & & & \\
\hline & & $R F+(n=609)$ & $446(73.23)$ & $146(23.97)$ & $17(2.79)$ & 14.78 & 0.271 & 0.999 & $0.86(0.66-1.13)$ \\
\hline & & SE- $(n=279)$ & $192(68.82)$ & $79(28.32)$ & $8(2.87)$ & 17.03 & & & \\
\hline & & $\mathrm{SE}+(\mathrm{n}=527)$ & $381(72.30)$ & $133(25.24)$ & $13(2.47)$ & 15.09 & 0.309 & 0.999 & $0.87(0.66-1.14)$ \\
\hline & & ACPA- $(n=317)$ & $228(71.92)$ & $85(26.81)$ & $4(1.26)$ & 14.67 & & & \\
\hline & & $\mathrm{ACPA}+(\mathrm{n}=462)$ & $326(70.56)$ & $120(25.97)$ & $16(3.46)$ & 16.45 & 0.343 & 0.999 & $1.15(0.87-1.52)$ \\
\hline \multirow[t]{6}{*}{ rs324987 } & $\mathrm{T} / \mathrm{C}$ & $R F-(n=280)$ & $100(35.71)$ & $134(47.86)$ & $46(16.43)$ & 40.36 & & & \\
\hline & & $R F+(n=591)$ & $214(36.21)$ & $276(46.70)$ & $101(17.09)$ & 40.44 & 0.974 & 0.999 & $1.00(0.82-1.23)$ \\
\hline & & SE- $(n=264)$ & $108(40.91)$ & $113(42.80)$ & $43(16.29)$ & 37.69 & & & \\
\hline & & $\mathrm{SE}+(\mathrm{n}=518)$ & $176(33.98)$ & $255(49.23)$ & $87(16.80)$ & 41.41 & 0.156 & 0.999 & $1.17(0.94-1.45)$ \\
\hline & & ACPA- $(n=296)$ & $120(40.54)$ & $132(44.59)$ & $44(14.86)$ & 37.16 & & & \\
\hline & & $\mathrm{ACPA}+(\mathrm{n}=459)$ & $154(33.55)$ & 228 (49.67) & $77(16.78)$ & 41.61 & 0.08467 & 0.999 & $1.21(0.97-1.49)$ \\
\hline \multirow[t]{6}{*}{ rs 10263447} & $\mathrm{G} / \mathrm{C}$ & $R F-(n=283)$ & $206(72.79)$ & $71(25.09)$ & $6(2.12)$ & 14.66 & & & \\
\hline & & $R F+(n=593)$ & $438(73.86)$ & $141(23.78)$ & $14(2.36)$ & 14.25 & 0.817 & 0.999 & $0.97(0.73-1.29)$ \\
\hline & & SE- $(n=266)$ & $203(76.32)$ & $60(22.56)$ & $3(1.13)$ & 12.41 & & & \\
\hline & & $\mathrm{SE}+(\mathrm{n}=520)$ & $387(74.42)$ & $120(23.08)$ & $13(2.50)$ & 14.04 & 0.370 & 0.999 & $1.15(0.84-1.58)$ \\
\hline & & ACPA- $(\mathrm{n}=299)$ & 229 (76.59) & $67(22.41)$ & $3(1.00)$ & 12.21 & & & \\
\hline & & $\mathrm{ACPA}+(\mathrm{n}=460)$ & $344(74.78)$ & $106(23.04)$ & $10(2.17)$ & 13.7 & 0.401 & 0.999 & $1.14(0.84-1.55)$ \\
\hline
\end{tabular}

*All p values have been calculated for the allelic model; **p values after Bonferroni correction; ***Odds ratio for the minor allele. RF+/-: positive/negative rheumatoid factor; $\mathrm{SE+/-:}$ positive/negative shared epitope; $\mathrm{ACPA}+/-:$ positive/negative anticitrullinated protein antibody; MAF: minor allele frequency. 
Table 3. Analysis of NPSR1 gene single nucleotide polymorphisms (SNP) for allelic combinations in Spanish patients with rheumatoid arthritis (RA) and healthy controls. Bold face values indicate statistical significance.

\begin{tabular}{lcccc}
\hline $\begin{array}{l}\text { Allelic } \\
\text { Combination }\end{array}$ & Controls, $\mathrm{n}(\%)$ & $\mathrm{RA}, \mathrm{n}(\%)$ & $\mathrm{p}$ & OR (95\% CI) \\
\hline CCACCCG & $105(10.4)$ & $87(6.9)$ & $\mathbf{0 . 0 0 3}$ & $0.64(0.48-0.87)$ \\
CCACCTG & $77(7.6)$ & $90(7.2)$ & 0.670 & $0.93(0.68-1.29)$ \\
CCACGCC & $167(16.6)$ & $188(15.0)$ & 0.300 & $0.89(0.71-1.12)$ \\
CGCCGCG & $120(11.9)$ & $186(14.8)$ & $\mathbf{0 . 0 4 4}$ & $1.28(1.00-1.67)$ \\
CGCCGTG & $185(18.4)$ & $211(17.0)$ & 0.336 & $0.90(0.72-1.12)$ \\
CGCTGTG & $352(35.0)$ & $491(38.7)$ & $\mathbf{0 . 0 4 4}$ & $1.28(1.00-1.64)$ \\
\hline
\end{tabular}

Order of the SNP: rs323917, rs323922, rs324377, rs324396, rs740347, rs324987, rs 10263447. Allelic combinations with a frequency lower than $5 \%$ in control groups were excluded from analysis.

8. Blakey JD, Sayers I, Ring SM, Strachan DP, Hall IP. Positionally cloned asthma susceptibility gene polymorphisms and disease risk in the British 1958 Birth Cohort. Thorax 2009;64:381-7.

9. Camilleri M, Carlson P, Zinsmeister AR, McKinzie S, Busciglio I, Burton D, et al. Neuropeptide s receptor induces neuropeptide expression and associates with intermediate phenotypes of functional gastrointestinal disorders. Gastroenterology 2009;138:98-107, e4.

10. D'Amato M, Zucchelli M, Seddighzadeh M, Anedda F, Lindblad S, Kere J, et al. Analysis of neuropeptide S receptor gene (NPSR1) polymorphism in rheumatoid arthritis. PLoS One 2010;5:e9315.

11. Arnett FC, Edworthy SM, Bloch DA, McShane DJ, Fries JF, Cooper NS, et al. The American Rheumatism Association 1987 revised criteria for the classification of rheumatoid arthritis. Arthritis Rheum 1988;31:315-24.
12. Holoshitz J. The rheumatoid arthritis HLA-DRB1 shared epitope. Curr Opin Rheumatol 2010;22:293-8.

13. Szodoray P, Szabo Z, Kapitany A, Gyetvai A, Lakos G, Szanto S, et al. Anti-citrullinated protein/peptide autoantibodies in association with genetic and environmental factors as indicators of disease outcome in rheumatoid arthritis. Autoimmun Rev 2010;9:140-3.

14. Kormann MS, Carr D, Klopp N, Illig T, Leupold W, Fritzsch C, et al. G-Protein-coupled receptor polymorphisms are associated with asthma in a large German population. Am J Respir Crit Care Med 2005; 171:1358-62.

15. Vergara C, Jimenez S, Acevedo N, Martinez B, Mercado D, Gusmao L, et al. Association of G-protein-coupled receptor 154 with asthma and total $\mathrm{IgE}$ in a population of the Caribbean coast of Colombia. Clin Exp Allergy 2009;39:1558-68.
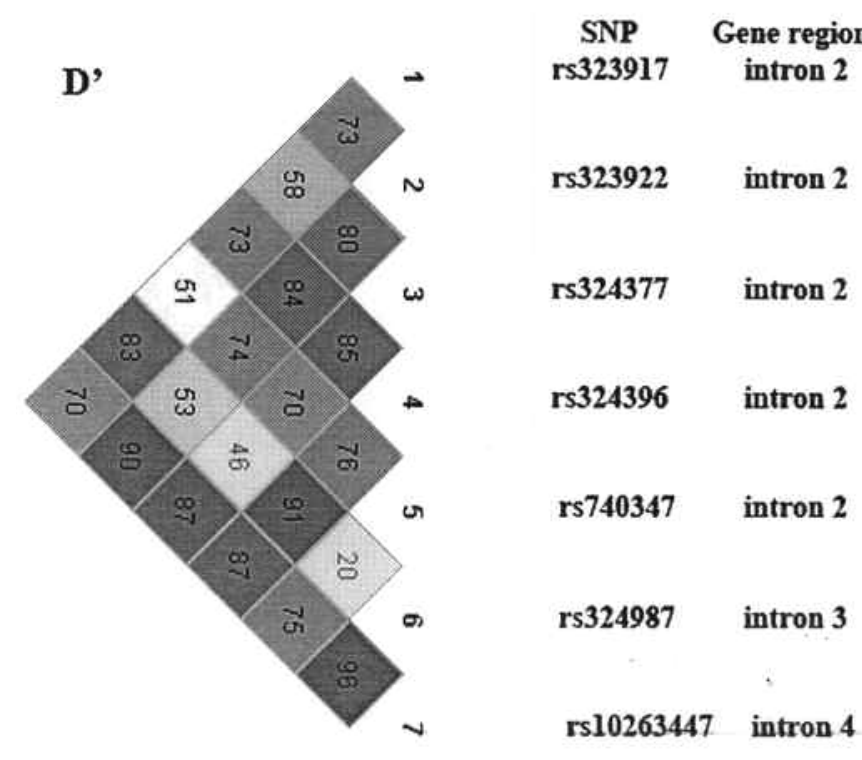

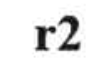

r2
SNP rs323917
Gene region intron 2

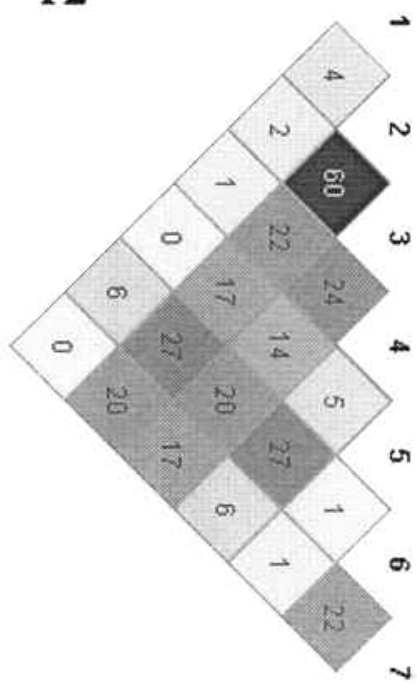

rs323922

intron 2

rs324377 intron 2

rs324396 intron 2

rs740347 intron 2

rs324987 intron 3

rs10263447 intron 4

\section{APPENDIX}

Linkage disequilibrium maps and location on the NPSR1 gene of the 7 studied variations as analyzed in Haploview software. Left panel: D' values; right panel: $\mathrm{r}^{2}$ values; right side of each panel: position of single-nucleotide polymorphism (SNP) in the gene. 